\title{
Mass spectra of heavy baryons
}

\section{Dietmar Ebert}

Institute of Physics, Humboldt University, Berlin, Germany

E-mail: debertephysik.hu-berlin.de

\section{Rudolf Faustov*}

Dorodnicyn Computing Centre, Russian Academy of Sciences, Moscow, Russia E-mail: faustoveccas.ru

\section{Vladimir Galkin ${ }^{\text {市 }}$}

Dorodnicyn Computing Centre, Russian Academy of Sciences, Moscow, Russia Institute of Physics, Humboldt University, Berlin, Germany

E-mail: galkinephysik.hu-berlin.de

Masses of the ground state and excited heavy baryons are calculated in the heavy-quark-lightdiquark picture within the relativistic quark model. The obtained predictions agree well with available experimental data.

8th Conference Quark Confinement and the Hadron Spectrum

September 1-6, 2008

Mainz. Germany

\footnotetext{
* Supported in part by the Russian Foundation for Basic Research (RFBR) grant No.08-02-00582

†Speaker.

¥Supported in part by the Deutscher Akademischer Austausch Dienst (DAAD), the Russian Science Support Foundation and the Russian Foundation for Basic Research (RFBR) grant No.08-02-00582
} 
During last few years a significant experimental progress has been achieved in studying heavy baryons with one heavy quark. At present masses of all ground states of charmed baryons as well as of their excitations are known experimentally with rather good precision [1]. Half of the ground state bottom baryon masses are also known now. Here we briefly review our studies of masses of the ground state and excited heavy baryons containing one heavy quark. All calculations [回, 解 are performed in the framework of the relativistic quark model based on the quasipotential approach in QCD. We use the heavy-quark-light-diquark approximation to reduce a complicated relativistic three-body problem to the subsequent solution of two more simple two-body problems.

In the considered model the diquark is described by the colour triplet wave function $\left(\Psi_{d}\right)$ of the two-quark bound state and the baryon is described by the wave function $\left(\Psi_{B}\right)$ of the quark-diquark bound state, satisfying the quasipotential equations of the Schrödinger type

$$
\left(\frac{b^{2}(M)}{2 \mu_{R}}-\frac{\mathbf{p}^{2}}{2 \mu_{R}}\right) \Psi_{d, B}(\mathbf{p})=\int \frac{d^{3} q}{(2 \pi)^{3}} V_{d, B}(\mathbf{p}, \mathbf{q} ; M) \Psi_{d, B}(\mathbf{q})
$$

where the relativistic reduced mass is

$$
\mu_{R}=\frac{M^{4}-\left(m_{1}^{2}-m_{2}^{2}\right)^{2}}{4 M^{3}}
$$

and the on-mass-shell relative momentum squared

$$
b^{2}(M)=\frac{\left[M^{2}-\left(m_{1}+m_{2}\right)^{2}\right]\left[M^{2}-\left(m_{1}-m_{2}\right)^{2}\right]}{4 M^{2}} .
$$

The kernel $V_{d, B}(\mathbf{p}, \mathbf{q} ; M)$ in Eq. (11) is the QCD motivated operator of the quark-quark $(d)$ or quark-diquark $(B)$ interaction. It is constructed with the help of the off-mass-shell scattering amplitude, projected onto the positive energy states. For the construction of the quark interaction potential in the diquark and baryon we closely follow the similar construction of the quark-antiquark interaction in mesons. For the quark-quark interaction in a diquark we use the relation $V_{q q}=V_{q \bar{q}} / 2$ arising under the assumption about the octet structure of the colour quark interaction. An important role in this construction is played by the Lorentz-structure of the nonperturbative confining interaction. In our analysis of mesons we adopted that the effective quark-antiquark interaction is the sum of the one-gluon exchange term and the mixture of long-range vector and scalar linear confining potentials with the vector confining potential containing the Pauli term. We use the same conventions for the construction of the quark-quark and quark-diquark interactions in the baryon. The explicit expressions for the quasipotential of the quark-quark $(q q)$ interaction in the diquark and quark-diquark $(Q d)$ interaction in the baryon are given in Refs. [2, 3]. Values of the model parameters can be also found in these references.

The first step is the calculation of masses, wave functions and form factors of the diquarks, composed from two relativistic light quarks. Next, at the second step, a heavy baryon is treated as a bound system of a relativistic light diquark and heavy quark. It is important to emphasize that we do not consider a diquark as a point particle but explicitly take into account its structure through the diquark-gluon vertex expressed in terms of the diquark wave functions. The calculated values of the ground state and excited baryon masses are given in Tables $1-5$ in comparison with available experimental data [1]. 


\begin{tabular}{|c|c|c|c|c|c|c|c|}
\hline \multirow{2}{*}{ Baryon } & \multirow[t]{2}{*}{$I\left(J^{P}\right)$} & \multicolumn{5}{|c|}{ Theory } & \multirow{2}{*}{$\begin{array}{c}\text { Experiment } \\
\text { PDG }\end{array}$} \\
\hline & & $\begin{array}{c}\text { our } \\
(2005)\end{array}$ & $\begin{array}{l}\text { Roberts } \\
\text { Pervin }\end{array}$ & $\begin{array}{c}\text { Karliner } \\
\text { et al }\end{array}$ & Jenkins & $\begin{array}{c}\text { Lewis } \\
\text { Woloshyn }\end{array}$ & \\
\hline$\Lambda_{c}$ & $0\left(\frac{1}{2}^{+}\right)$ & 2297 & 2268 & & & & $2286.46(14)$ \\
\hline$\Sigma_{c}$ & $1\left(\frac{1}{2}^{+}\right)$ & 2439 & 2455 & & & & $2453.76(18)$ \\
\hline$\Sigma_{c}^{*}$ & $1\left(\frac{3}{2}^{+}\right)$ & 2518 & 2519 & & & & $2518.0(5)$ \\
\hline$\Xi_{c}$ & $\left.\frac{1}{2}_{2} \frac{1}{2}^{+}\right)$ & 2481 & 2466 & & & & $2471.0(4)$ \\
\hline$\Xi_{c}^{\prime}$ & $\left.\frac{1}{2}_{2} \frac{1}{2}^{+}\right)$ & 2578 & 2594 & & $2580.8(2.1)$ & & $2578.0(2.9)$ \\
\hline$\Xi_{c}^{*}$ & $\frac{1}{2}\left(\frac{3}{2}^{+}\right)$ & 2654 & 2649 & & & & $2646.1(1.2)$ \\
\hline$\Omega_{c}$ & $0\left(\frac{1}{2}^{+}\right)$ & 2698 & 2718 & & & & $2697.5(2.6)$ \\
\hline$\Omega_{c}^{*}$ & $0\left(\frac{3}{2}^{+}\right)$ & 2768 & 2776 & & $2760.5(4.9)$ & & $2768.3(3.0)^{\dagger}$ \\
\hline$\Lambda_{b}$ & $0\left(\frac{1}{2}^{+}\right)$ & 5622 & 5612 & & & $5628\left(\begin{array}{c}23 \\
50\end{array}\right)$ & $5620.2(1.6)$ \\
\hline$\Sigma_{b}$ & $1\left(\frac{1}{2}^{+}\right)$ & 5805 & 5833 & 5814 & $5824.2(9.0)$ & $5793\left(\begin{array}{l}17 \\
21\end{array}\right)$ & $5807.5(2.5)^{\ddagger}$ \\
\hline$\Sigma_{b}^{*}$ & $1\left(\frac{3}{2}^{+}\right)$ & 5834 & 5858 & 5836 & $5840.0(8.8)$ & $5814\left(\begin{array}{l}26 \\
27\end{array}\right)$ & $5829.0(2.3)^{\ddagger}$ \\
\hline$\Xi_{b}$ & $\left.\frac{1}{2}^{2} \frac{1}{2}^{+}\right)$ & 5812 & 5806 & $5795(5)$ & $5805.7(8.1)$ & $5755\left(\begin{array}{l}18 \\
23\end{array}\right)$ & $5792.9(3.0)^{\star}$ \\
\hline$\Xi_{b}^{\prime}$ & $\frac{1}{2}\left(\frac{1}{2}^{+}\right)$ & 5937 & 5970 & $5930(5)$ & $5950.9(8.5)$ & $5885\left(\begin{array}{l}15 \\
18\end{array}\right)$ & \\
\hline$\Xi_{b}^{*}$ & $\frac{1}{2}_{2}\left(\frac{3}{2}^{+}\right)$ & 5963 & 5980 & $5959(4)$ & 5966.1(8.3) & $5897\left(\begin{array}{l}40 \\
25\end{array}\right)$ & \\
\hline$\Omega_{b}$ & $0\left(\frac{1}{2}^{+}\right)$ & 6065 & 6081 & $6052(6)$ & $6068.7(11.1)$ & $6001\left(\begin{array}{l}12 \\
19\end{array}\right)$ & $6165(23)^{\star \star}$ \\
\hline$\Omega_{b}^{*}$ & $0\left(\frac{3}{2}^{+}\right)$ & 6088 & 6102 & $6083(6)$ & $6083.2(11.0)$ & $6013\left(\begin{array}{c}18 \\
23\end{array}\right)$ & \\
\hline
\end{tabular}

${ }^{\dagger} \mathrm{BaBar} 2006 ; \quad{ }^{\ddagger} \mathrm{CDF} 2006\left(\Sigma_{b}^{+}\right) ;{ }^{\star} \mathrm{CDF} 2007 ; \quad{ }^{\star} \mathrm{D} 02008$

Table 1: Comparison of theoretical predictions and experimental data for masses of the ground state heavy baryons (in $\mathrm{MeV}$ ).

\begin{tabular}{cccccccc}
\hline & & \multicolumn{2}{c}{$Q=c$} & & \multicolumn{2}{c}{$Q=b$} \\
\cline { 3 - 4 } \cline { 6 - 7 }$I\left(J^{P}\right)$ & $Q d$ state & $M$ & $M^{\exp }$ [1] & & $M$ & $M^{\exp }[1]$ & $M^{\exp } \mathrm{CDF}$ \\
\hline $0\left(\frac{1}{2}^{+}\right)$ & $1 S$ & 2297 & $2286.46(14)$ & 5622 & $5624(9)$ & $5619.7(2.4)$ \\
$0\left(\frac{1}{2}^{-}\right)$ & $1 P$ & 2598 & $2595.4(6)$ & 5930 & & \\
$0\left(\frac{3}{2}^{-}\right)$ & $1 P$ & 2628 & $2628.1(6)$ & 5947 & & \\
$0\left(\frac{1}{2}^{+}\right)$ & $2 S$ & 2772 & $2766.6(2.4) ?$ & 6086 & & \\
$0\left(\frac{3}{2}^{+}\right)$ & $1 D$ & 2874 & & & 6189 & & \\
$0\left(\frac{5}{2}^{+}\right)$ & $1 D$ & 2883 & $2882.5(2.2) ?$ & 6197 & & \\
\hline
\end{tabular}

Table 2: Masses of the $\Lambda_{Q}(Q=c, b)$ heavy baryons (in $\mathrm{MeV}$ ).

The mass spectra of the $\Lambda_{Q}$ and $\Sigma_{Q}$ baryons are presented in Tables 2, 3. Masses of the ground states are measured both for charmed and bottom $\Lambda_{Q}$ and $\Sigma_{Q}$ baryons. For charmed baryons the masses of several excited states are also known. It is important to emphasize that the $J^{P}$ quantum numbers for most excited heavy baryons have not been determined experimentally, but are assigned by PDG on the basis of quark model predictions. For some excited charm baryons such as the $\Lambda_{c}(2765), \Lambda_{c}(2880)$ and $\Lambda_{c}(2940)$ it is even not known if they are excitations of the $\Lambda_{c}$ or $\Sigma_{c}$. Our calculations show that the $\Lambda_{c}$ (2765) can be either the first radial (2S) excitation of the $\Lambda_{c}$ with $J^{P}=\frac{1}{2}^{+}$containing the light scalar diquark or the first orbital excitation $(1 P)$ of the $\Sigma_{c}$ with $J^{P}=\frac{3}{2}^{-}$ 


\begin{tabular}{|c|c|c|c|c|c|c|c|}
\hline \multirow[b]{2}{*}{$I\left(J^{P}\right)$} & \multirow[b]{2}{*}{$Q d$ state } & \multicolumn{4}{|c|}{$Q=c$} & \multicolumn{2}{|r|}{$Q=b$} \\
\hline & & $M$ & $M^{\exp }$ [1]] & $M^{\exp } \mathrm{BaBar}$ & $M^{\exp }$ Belle & $M$ & $M^{\exp } \mathrm{CDF}$ \\
\hline $1\left(\frac{1}{2}^{+}\right)$ & $1 S$ & 2439 & $2453.76(18)$ & & & 5805 & $5807.5(2.6)$ \\
\hline $1\left(\frac{3}{2}^{+}\right)$ & $1 S$ & 2518 & $2518.0(5)$ & & & 5834 & $5829.0(2.4)$ \\
\hline $1\left(\frac{1}{2}^{-}\right)$ & $1 P$ & 2805 & & & & 6122 & \\
\hline $1\left(\frac{1}{2}^{-}\right)$ & $1 P$ & 2795 & & & & 6108 & \\
\hline $1\left(\frac{3}{2}^{-}\right)$ & $1 P$ & 2799 & $2802\left({ }_{7}^{4}\right)$ & & & 6106 & \\
\hline $1\left(\frac{3}{2}^{-}\right)$ & $1 P$ & 2761 & $2766.6(2.4) ?$ & & & 6076 & \\
\hline $1\left(\frac{5}{2}^{-}\right)$ & $1 P$ & 2790 & & & & 6083 & \\
\hline $1\left(\frac{1}{2}^{+}\right)$ & $2 S$ & 2864 & & & & 6202 & \\
\hline $1\left(\frac{3}{2}^{+}\right)$ & $2 S$ & 2912 & & 2939.8(2.3)? & $2938\left(\frac{3}{5}\right) ?$ & 6222 & \\
\hline
\end{tabular}

Table 3: Masses of the $\Sigma_{Q}(Q=c, b)$ heavy baryons (in $\mathrm{MeV}$ ).

\begin{tabular}{|c|c|c|c|c|c|c|}
\hline \multirow[b]{2}{*}{$I\left(J^{P}\right)$} & \multirow[b]{2}{*}{$Q d$ state } & \multicolumn{3}{|c|}{$Q=c$} & \multicolumn{2}{|r|}{$Q=b$} \\
\hline & & $M$ & $M^{\exp }$ [1] & $M^{\exp } \mathrm{BaBar}$ & $M$ & $M^{\exp } \mathrm{CDF}$ \\
\hline$\left.\frac{1}{2}^{(} \frac{1}{2}^{+}\right)$ & $1 S$ & 2481 & $2471.0(4)$ & & 5812 & $5792.9(3.0)$ \\
\hline & $1 P$ & 2801 & $2791.9(3.3)$ & & 6119 & \\
\hline$\frac{1}{2}\left(\frac{3}{2}^{-}\right)$ & $1 P$ & 2820 & $2818.2(2.1)$ & & 6130 & \\
\hline$\frac{1}{2}\left(\frac{1}{2}^{+}\right)$ & $2 S$ & 2923 & & & 6264 & \\
\hline$\frac{1}{2}\left(\frac{3}{2}^{+}\right)$ & $1 D$ & 3030 & & & 6359 & \\
\hline$\left.\frac{1}{2}^{\left(\frac{5}{2}\right.}{ }^{+}\right)$ & $1 D$ & 3042 & & $3054.2(1.5)$ & 6365 & \\
\hline
\end{tabular}

Table 4: Masses of the $\Xi_{Q}(Q=c, b)$ heavy baryons with a scalar diquark (in MeV).

containing the light axial vector diquark. The $\Lambda_{c}(2880)$ baryon in our model is well described by the second orbital $(1 D)$ excitation of the $\Lambda_{c}$ with $J^{P}=\frac{5}{2}^{+}$in agreement with the recent spin assignment by Belle based on the analysis of angular distributions in the decays $\Lambda_{c}(2880)^{+} \rightarrow$ $\Sigma_{c}(2455)^{0,++} \pi^{+,-}$. Our model suggests that the charmed baryon $\Lambda_{c}(2940)$, recently discovered by BaBar and then also confirmed by Belle, could be the first radial $(2 S)$ excitation of the $\Sigma_{c}$ with $J^{P}=\frac{3}{2}^{+}$which mass is predicted slightly below the experimental value. The $\Sigma_{c}(2800)$ baryon can be identified in our model with one of the orbital (1P) excitations of the $\Sigma_{c}$ with $J^{P}=\frac{1}{2}^{-}, \frac{3}{2}^{-}$or $\frac{5}{2}^{-}$which predicted mass differences are less than $15 \mathrm{MeV}$. Thus masses of all these states are compatible with the experimental values within errors.

Mass spectra of the $\Xi_{Q}$ baryons with the scalar and axial vector light ( $q s$ ) diquarks are given in Tables 4, 5. Our model prediction for the $M_{\Xi_{b}}$ is in a reasonable agreement with recently obtained data. In the excited charmed baryon sector we can identify the $\Xi_{c}(2790)$ and $\Xi_{c}(2815)$ with the first orbital (1P) excitations of the $\Xi_{c}$ with $J^{P}=\frac{1}{2}^{-}$and $J^{P}=\frac{3}{2}^{-}$, respectively, containing the light scalar diquark, which is in agreement with the PDG [1] assignment. Recently Belle observed two baryons $\Xi_{c}(2980)$ and $\Xi_{c}(3077)$, which existence was also confirmed by BaBar. The $\Xi_{c}(2980)$ can be interpreted in our model as the first radial $(2 S)$ excitation of the $\Xi_{c}$ with $J^{P}=\frac{1}{2}^{+}$containing the light axial vector diquark. On the other hand the $\Xi_{c}(3077)$ corresponds to the second orbital $(1 D)$ excitation in this system with $J^{P}=\frac{5}{2}^{+}$. Very recently BaBar observed two new charmed baryons 


\begin{tabular}{|c|c|c|c|c|c|c|}
\hline \multirow[b]{2}{*}{$I\left(J^{P}\right)$} & \multirow[b]{2}{*}{$Q d$ state } & \multicolumn{4}{|c|}{$Q=c$} & \multirow{2}{*}{$\begin{array}{c}Q=b \\
M\end{array}$} \\
\hline & & $M$ & $M^{\exp }$ [1] & $M^{\exp }$ Belle & $M^{\exp } \mathrm{BaBar}$ & \\
\hline$\left.{ }^{+}\right)$ & $1 S$ & 2578 & $2578.0(2.9)$ & & & 5937 \\
\hline & $1 S$ & 2654 & $2646.1(1.2)$ & & & 5963 \\
\hline- ) & $1 P$ & 2934 & & & & 6249 \\
\hline$\frac{1}{2}_{2}\left(\frac{1}{2}^{-}\right)$ & $1 P$ & 2928 & & & & 6238 \\
\hline & $1 P$ & 2931 & & & & 6237 \\
\hline- ) & $1 P$ & 2900 & & & & 6212 \\
\hline$\frac{1}{2}\left(\frac{5}{2}^{-}\right)$ & $1 P$ & 2921 & & & & 6218 \\
\hline$\frac{1}{2}\left(\frac{1}{2}^{+}\right)$ & $2 S$ & 2984 & & $2978.5(4.1)$ & 2967.1(2.9) & 6327 \\
\hline & $2 S$ & 3035 & & & & 6341 \\
\hline$\left.\frac{1}{2}_{2} \frac{1}{2}^{+}\right)$ & $1 D$ & 3132 & & & & 6420 \\
\hline$\frac{1}{2}\left(\frac{3}{2}^{+}\right)$ & $1 D$ & 3127 & & & & 6410 \\
\hline$\frac{1}{2}_{2}\left(\frac{3}{2}^{+}\right)$ & $1 D$ & 3131 & & & & 6412 \\
\hline$\frac{1}{2}\left(\frac{5}{2}^{+}\right)$ & $1 D$ & 3123 & & & $3122.9(1.4)$ & 6403 \\
\hline$\frac{1}{2}\left(\frac{5}{2}^{+}\right)$ & $1 D$ & 3087 & & $3082.8(3.3)$ & $3076.4(1.0)$ & 6377 \\
\hline$\frac{1}{2}\left(\frac{7}{2}^{+}\right)$ & $1 D$ & 3136 & & & & 6390 \\
\hline
\end{tabular}

Table 5: Masses of the $\Xi_{Q}(Q=c, b)$ heavy baryons with an axial vector diquark (in $\mathrm{MeV}$ ).

$\Xi_{c}(3055)$ and $\Xi_{c}(3123)$. These states can be interpreted in our model as the second orbital $(1 D)$ excitations of the $\Xi_{c}$ with $J^{P}=\frac{5}{2}^{+}$containing scalar and axial vector diquarks, respectively.

For the $\Omega_{Q}$ baryons up till very recently only masses of the ground-state charmed baryons were known. In 2006 the $\Omega_{c}^{*}$ baryon was discovered by BaBar. The measured mass difference of the $\Omega_{c}^{*}$ and $\Omega_{c}$ baryons of $(70.8 \pm 1.0 \pm 1.1) \mathrm{MeV}$ is in very good agreement with the prediction of our model $70 \mathrm{MeV}$ [2]. Just before this conference D0 reported the observation of the $\Omega_{b}$ baryon with the mass of $6.165 \pm 0.010 \pm 0.013 \mathrm{GeV}$ 㺻. This value is somewhat higher than almost all theoretical predictions (including ours $M_{\Omega_{b}}=6065 \mathrm{MeV}$ ). However the additional experimental confirmation is needed.

In summary, we find that all presently available experimental data for the masses of the ground state and excited heavy baryons can be accommodated in the picture treating a heavy baryon as the composite system of a light diquark and heavy quark, experiencing orbital and radial excitations only between these constituents.

\section{References}

[1] C. Amsler et al. [Particle Data Group], Review of particle physics, Phys. Lett. B 667, 1 (2008).

[2] D. Ebert, R. N. Faustov and V. O. Galkin, Masses of heavy baryons in the relativistic quark model, Phys. Rev. D 72, 034026 (2005) [arXiv:hep-ph/0504112].

[3] D. Ebert, R. N. Faustov and V. O. Galkin, Masses of excited heavy baryons in the relativistic quark model, Phys. Lett. B 659, 612 (2008) [arXiv:0705.2957 [hep-ph]].

[4] V. M. Abazov et al. [D0 Collaboration], Observation of the doubly strange b baryon $\Omega_{b}^{-}$, arXiv:0808.4142 [hep-ex]. 